Supporting Information for

\title{
Upconverting Oil-Laden Hollow Mesoporous Silica Microcapsules for Anti-Stokes-Based Biophotonic Applications
}

Hak-Lae Lee ${ }^{\dagger}$, Jung Hwan Park ${ }^{\dagger}$,Hyun-Seok Choe ${ }^{\dagger}$, Myung-Soo Lee ${ }^{\dagger}$, Jeong-Min Park ${ }^{\dagger}$, Naoyuki Haradall, Yoichi Sasakill, Nobuhiro Yanai ${ }^{\prime}, \perp$, Nobuo Kimizukall, Jintao Zhu ${ }^{\S}$, Suk Ho Bhang:*, and Jae-Hyuk Kim ${ }^{\dagger} *$ *

${ }^{\dagger}$ Department of Chemical and Environmental Engineering, Pusan National University, Busan 46241, Korea

School of Chemical Engineering, Sungkyunkwan University, Suwon 440-746, Korea

$\S$ School of Chemistry and Chemical Engineering, Huazhong University of Science and Technology, Wuhan 430074, China

\| Department of Chemistry and Biochemistry, Graduate School of Engineering, Center for Molecular Systems (CMS), Kyushu University, 744 Moto-oka, Nishi-ku, Fukuoka 819-0395, Japan

${ }^{\perp}$ PRESTO, JST, Honcho 4-1-8, Kawaguchi, Saitama 332-0012, Japan

*Email: jaehyuk.kim@pusan.ac.kr; sukhobhang@skku.edu 

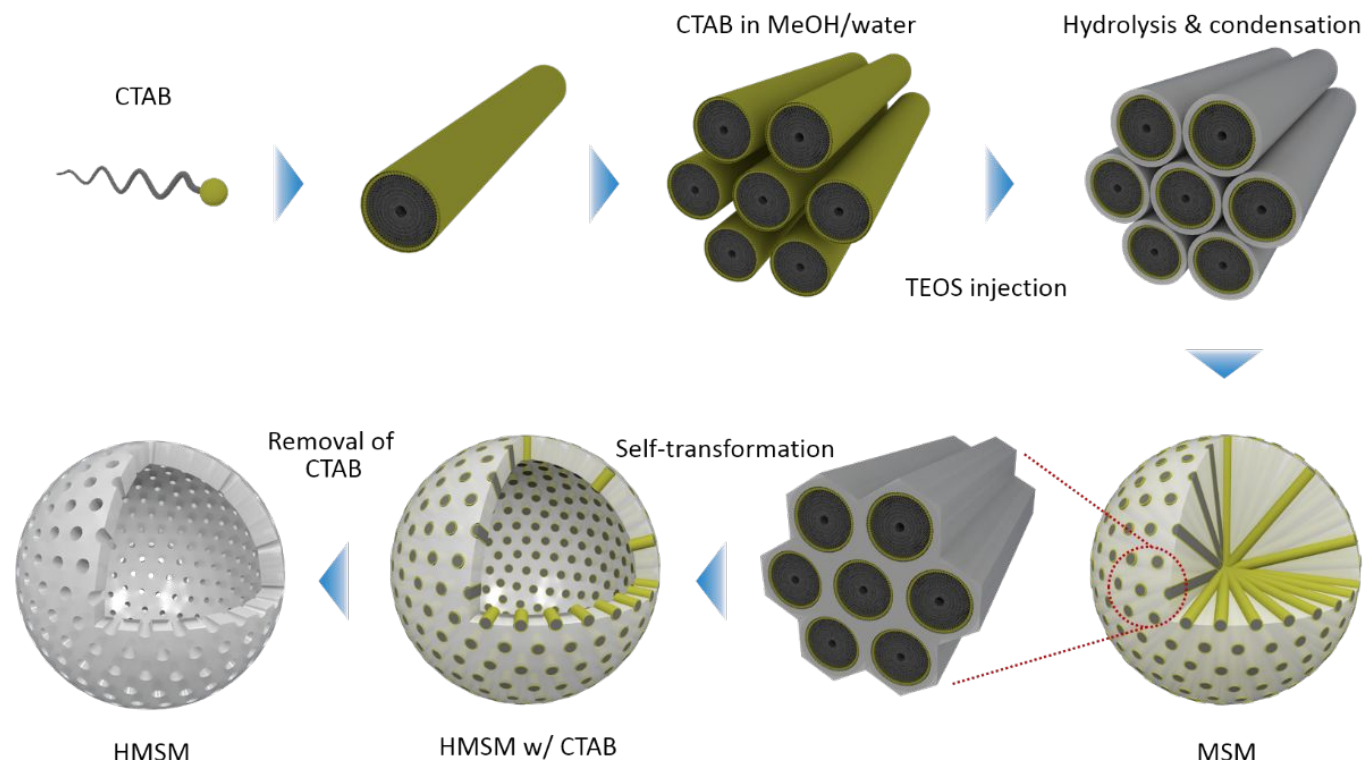

Figure S1. Schematic of the synthesis of HMSM based on a surfactant-assisted Stöber method in methanol/water solution and a subsequent self-transformation approach.
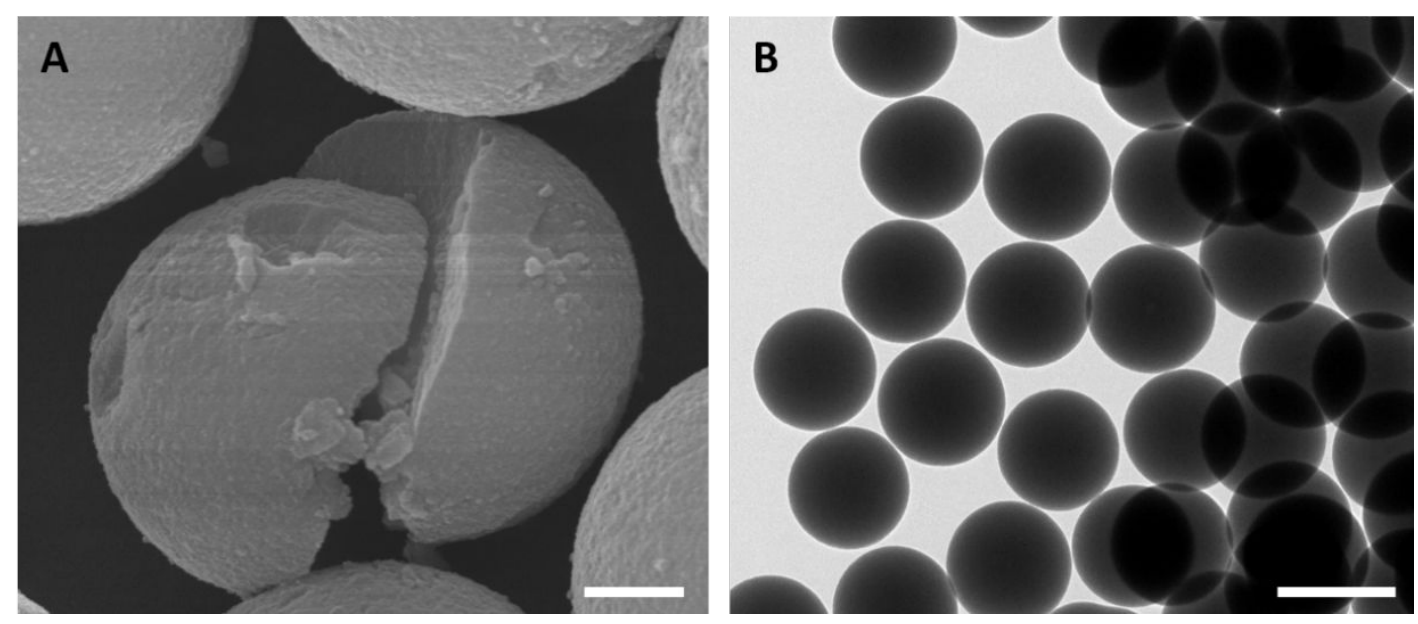

Figure S2. A) SEM and B) TEM images of MSMs synthesized in a methanol/water solution. Scale bars in (A) and (B) represent $250 \mathrm{~nm}$ and $1 \mu \mathrm{m}$, respectively. 



Figure S3. Low-magnification SEM images of HMSMs obtained with various amounts of deionized water: A) 10.7, B) 9.2, and C) $7.7 \mathrm{~mL}$. Scale bars represent $3 \mu \mathrm{m}$.

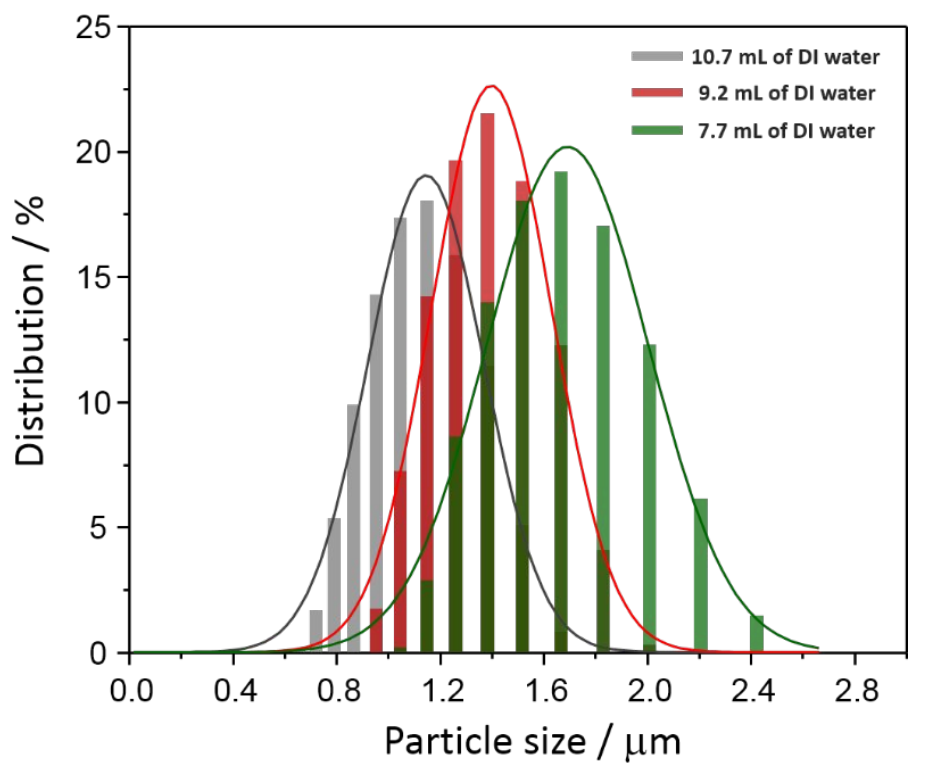

Figure S4. Size distributions of HMSMs dispersed in ethanol. 


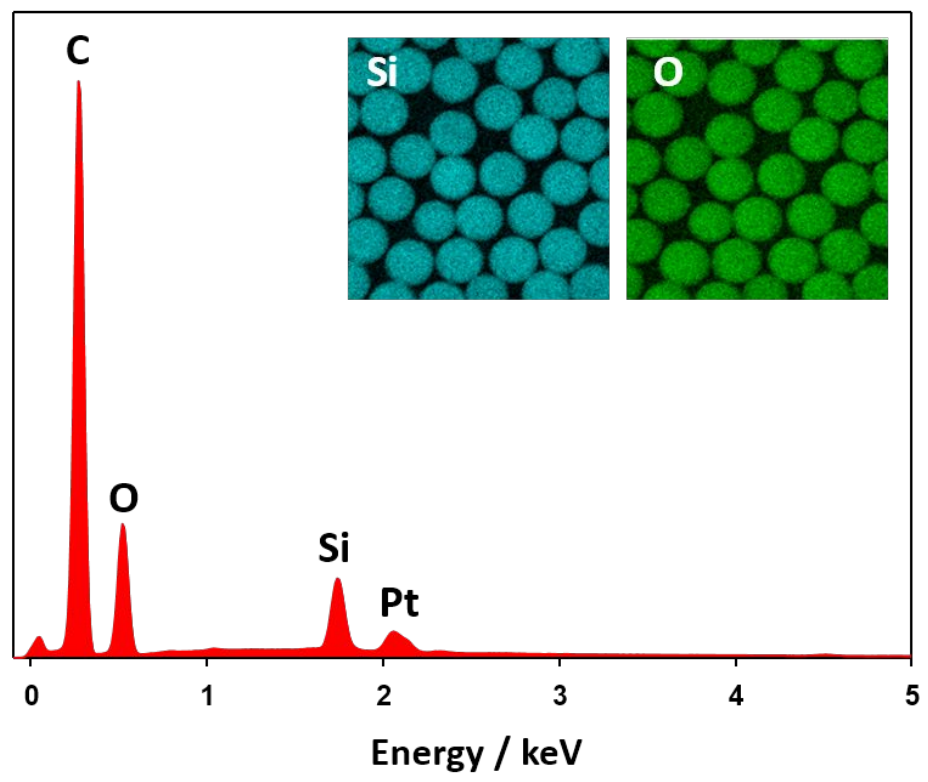

Figure S5. EDX elemental maps of HMSMs.

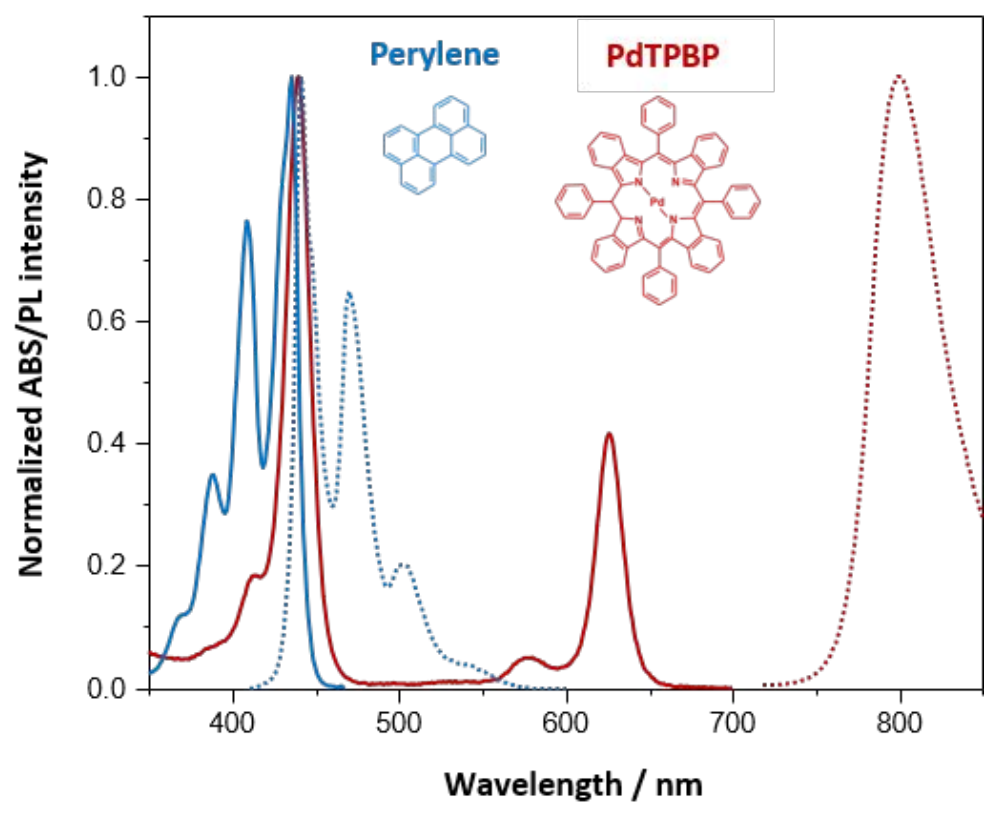

Figure S6. Molecular structure and normalized absorption (solid) and emission (dashed) spectra of perylene (blue) and PdTPBP (red) in oleic acid. 


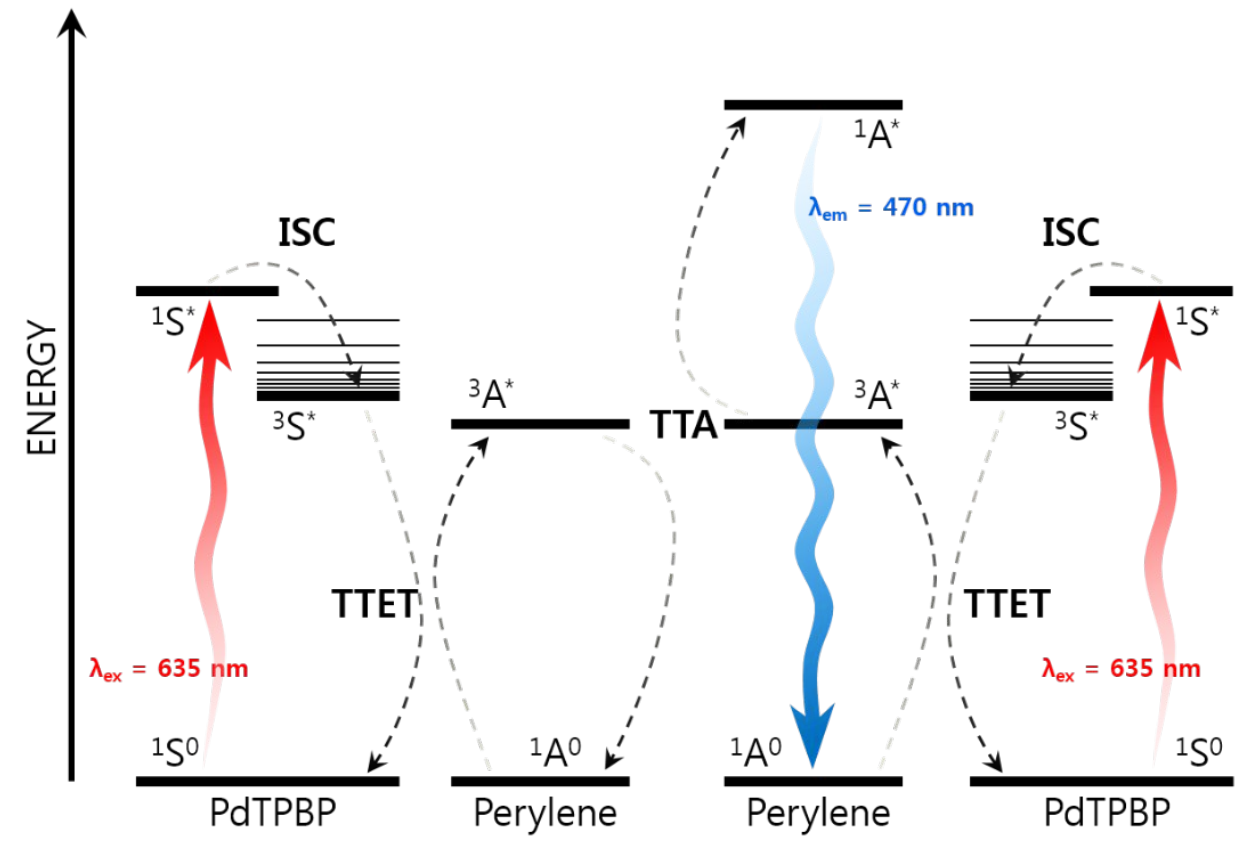

Figure S7. Jablonski diagram of the photochemical processes of TTA-UC; absorption (635 $\mathrm{nm}$ ), intersystem crossing (ISC), triplet-triplet energy transfer (TTET), triplet-triplet annihilation (TTA), and emission $(470 \mathrm{~nm})$.

A

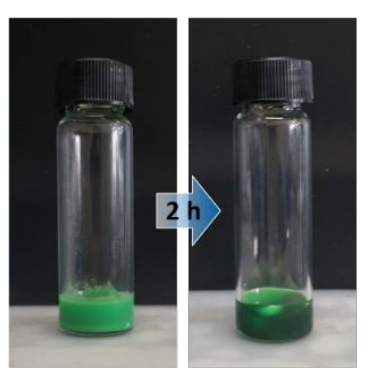

B

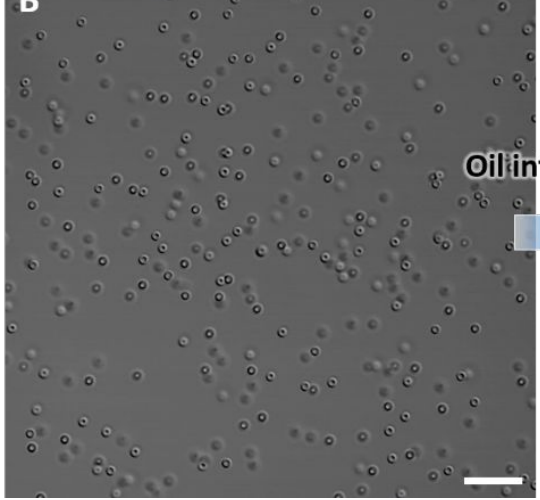

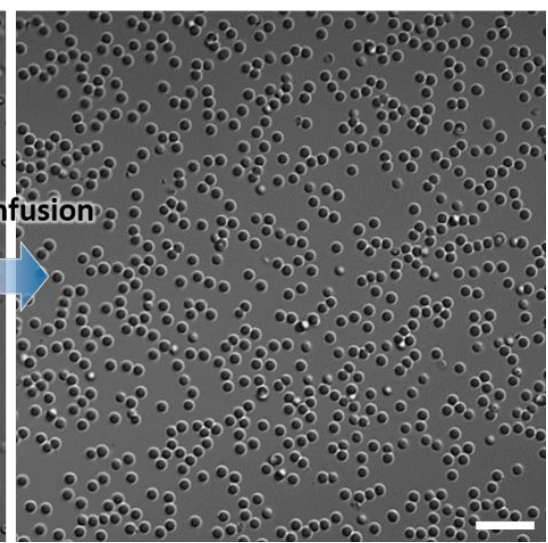

Figure S8. A) Ditigal images showing the post-encapsulation of the bulk UC oil into HMSMs under magnetic stirring. B) Bright-field images of HMSMs before (left) and after (right) the encapsulation of the UC oil. Scale bars in (B) represent $10 \mu \mathrm{m}$. 


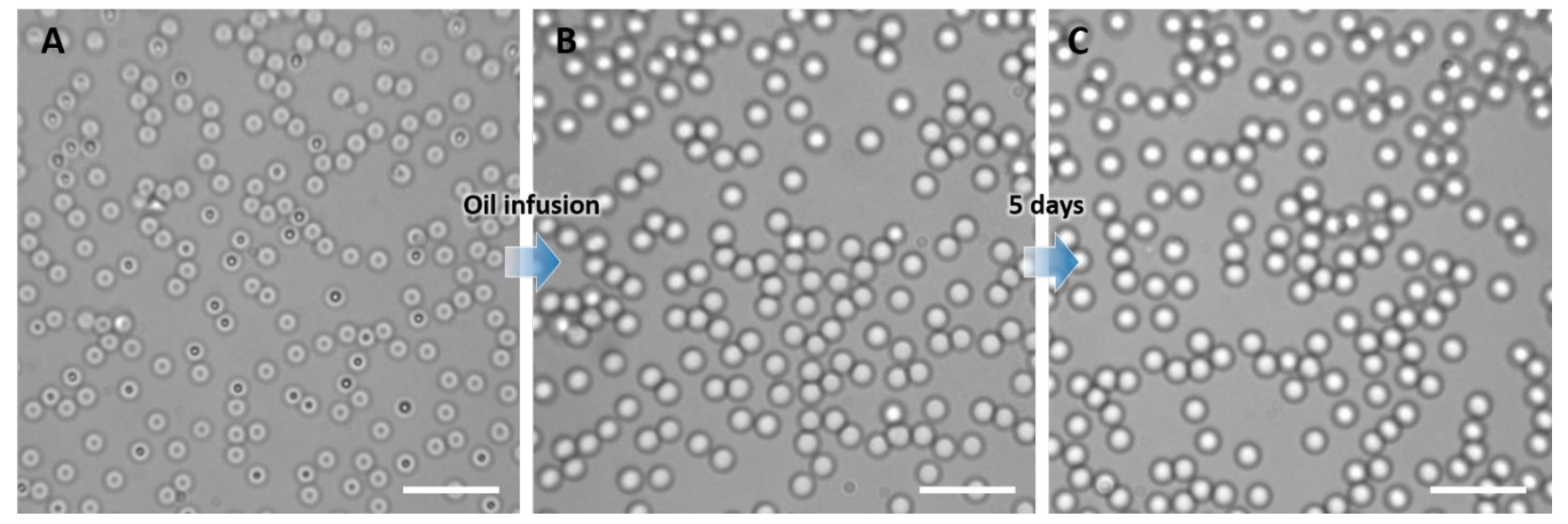

Figure S9. Microscopic images of the water suspension of A) HMSMs, B) UC-HMSMs, and C) UC-HMSMs after stored for 5 days. Scale bars represent $10 \mu \mathrm{m}$.
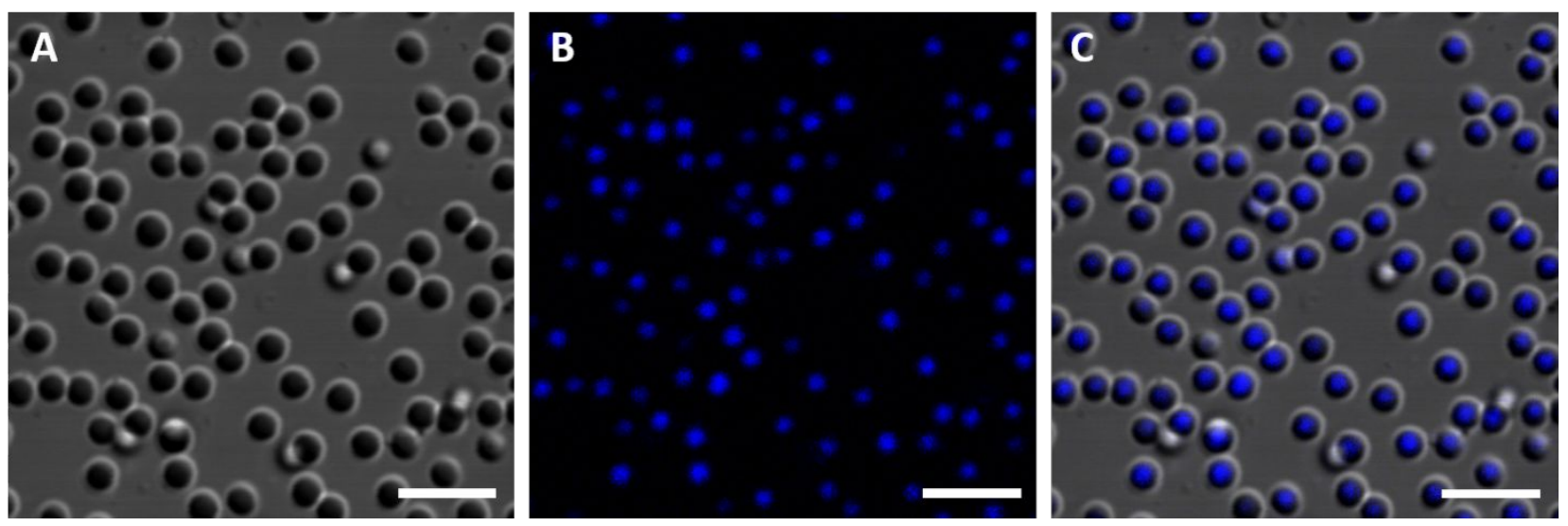

Figure S10. CLSM images of the water suspension of UC-HMSMs ([PdTPBP] $=0.43 \mathrm{mM}$ and [perylene] $=39.6 \mathrm{mM}$ in encapsulated OA) under $640 \mathrm{~nm}$ laser excitation; A) bright-field, B) fluorescence channel (450-550 nm), and C) merged image. Scale bars represent $5 \mu \mathrm{m}$. 


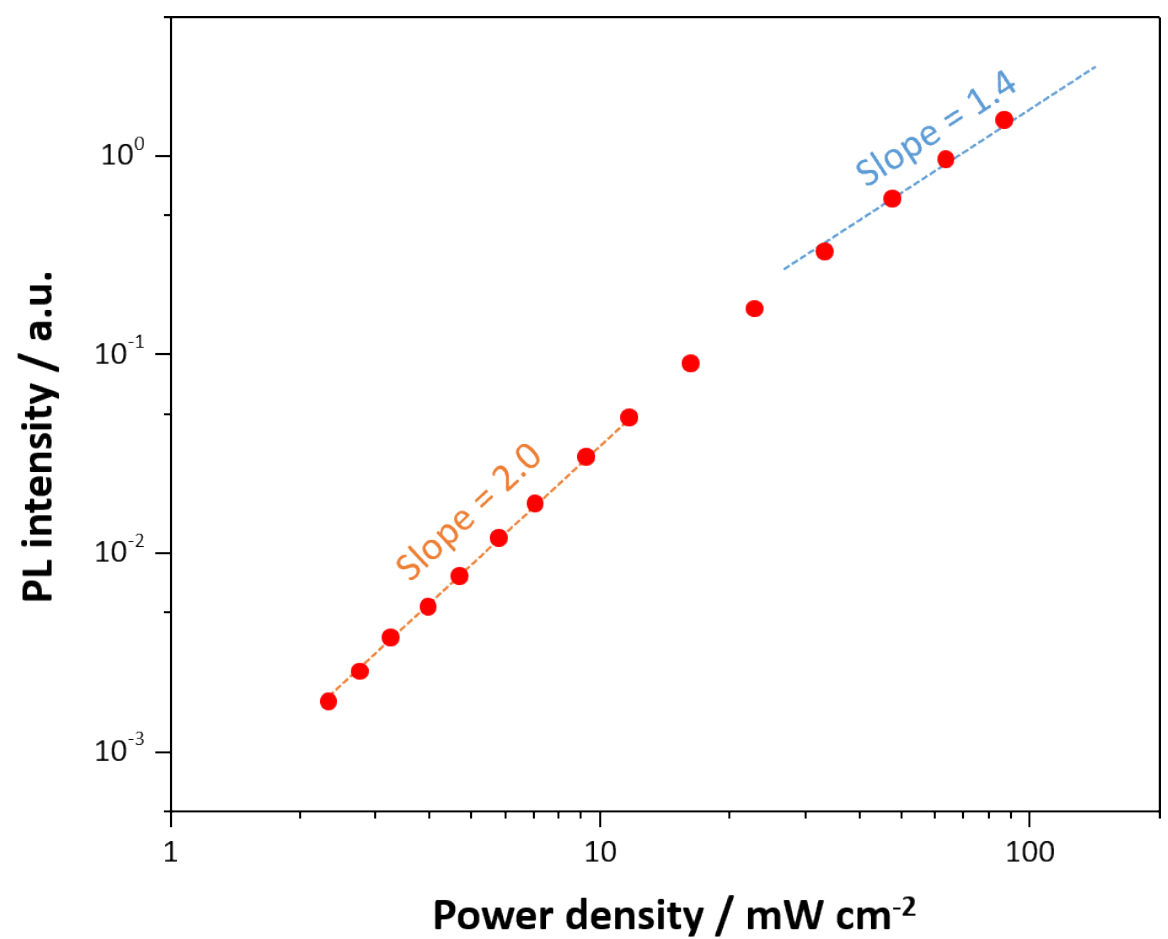

Figure S11. UC emission intensity $(470 \mathrm{~nm})$ from the UC-HMSMs aqueous suspension $([\mathrm{PdTPBP}]=0.43 \mathrm{mM}$ and $[$ perylene $]=39.6 \mathrm{mM}$ in encapsulated OA $)$ as a function of excitation power density under $635 \mathrm{~nm}$ laser irradiation.

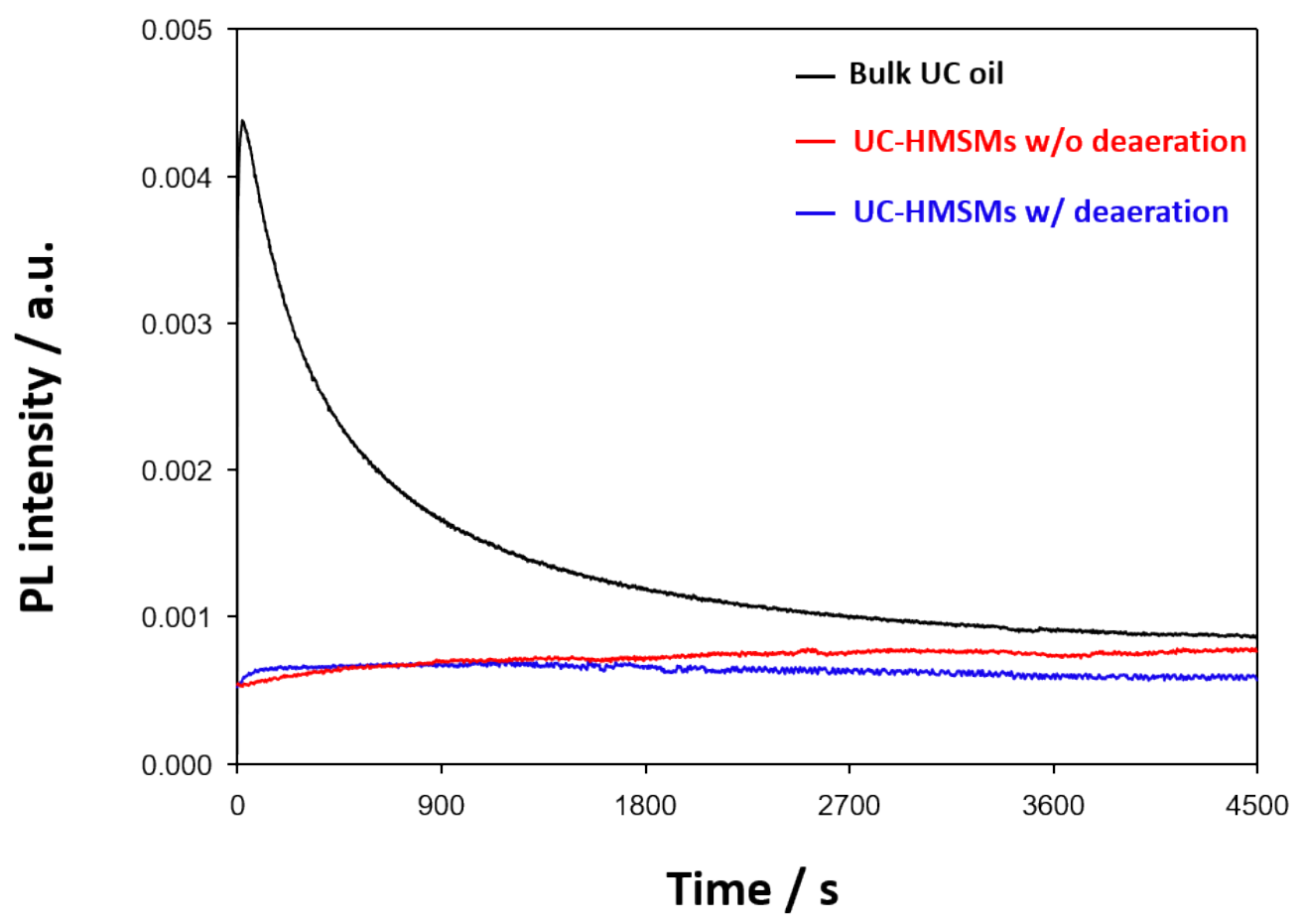

Figure S12. Stability of UC emission ( $470 \mathrm{~nm}$ ) from the bulk UC oil and UC-HMSMs aqueous suspensions $([\mathrm{PdTPBP}]=0.43 \mathrm{mM}$ and [perylene $]=39.6 \mathrm{mM}$ in encapsulated $\mathrm{OA})$ with and without deaeration under continuous $66.6 \mathrm{~mW} \mathrm{~cm}^{-2}$ laser excitation at $635 \mathrm{~nm}$. 


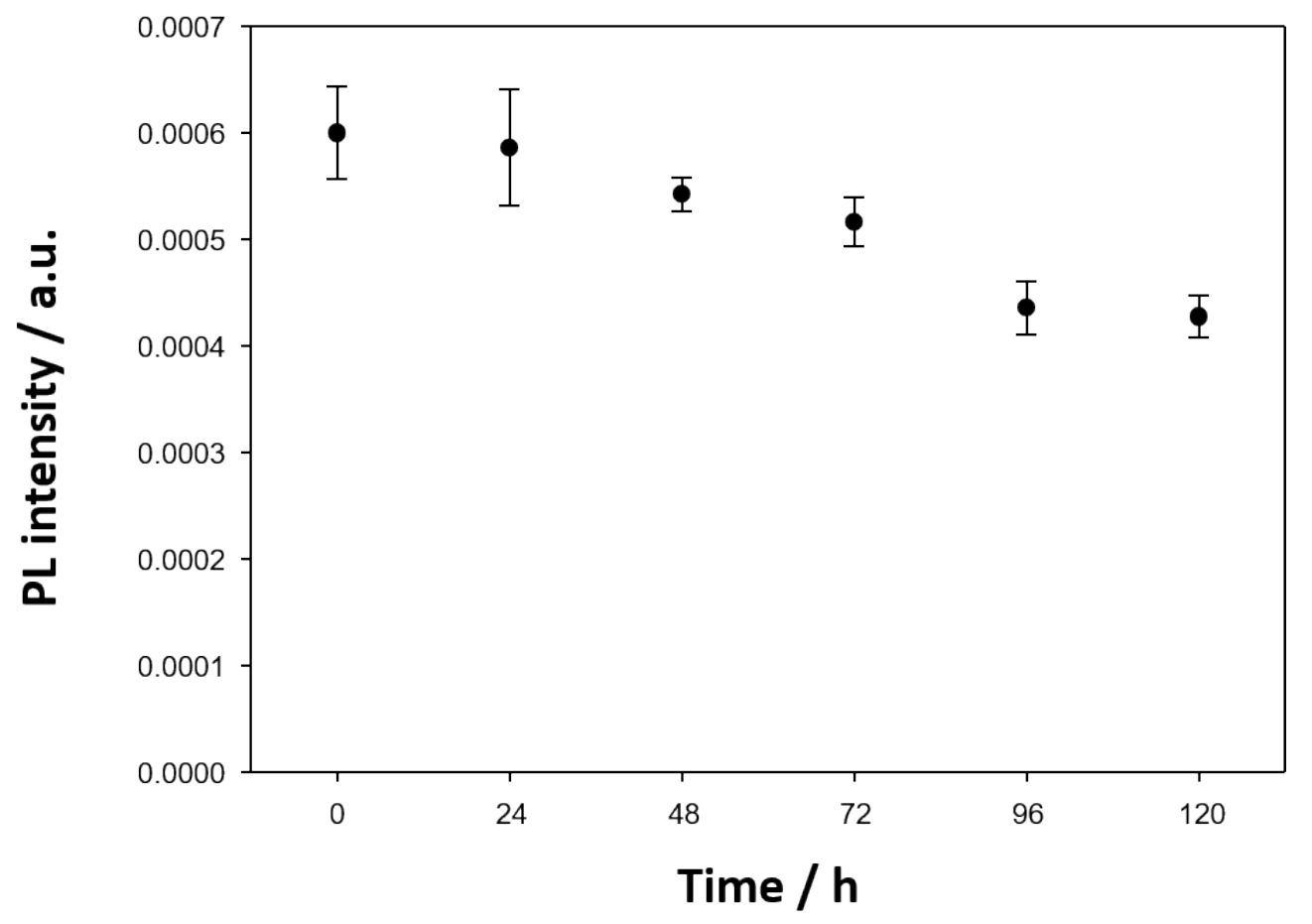

Figure S13. Shelf-life of the UC-HMSMs aqueous suspension ([PdTPBP] $=0.43 \mathrm{mM}$ and [perylene] $=39.6 \mathrm{mM}$ in encapsulated OA). The sample was stored in a dark room and the UC emission intensity $(470 \mathrm{~nm})$ was recorded every $24 \mathrm{~h}$ using $66.6 \mathrm{~mW} \mathrm{~cm}^{-2}$ laser excitation at $635 \mathrm{~nm}$.

A
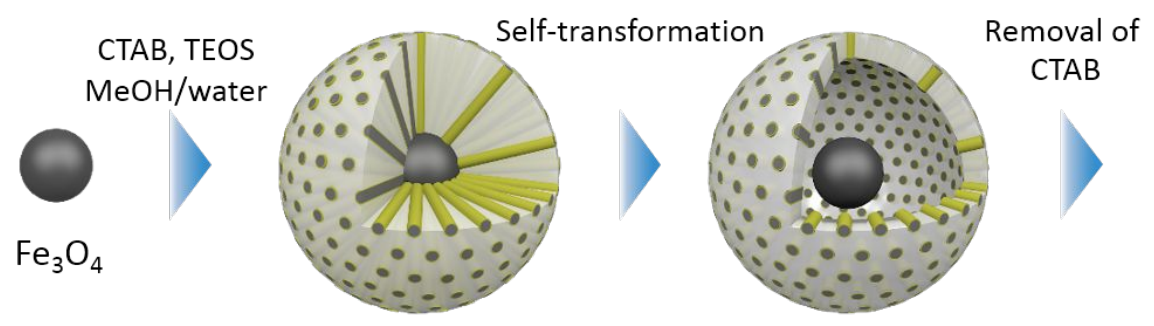

$$
\mathrm{Fe}_{3} \mathrm{O}_{4} @ \mathrm{mSiO} \mathrm{S}_{2}
$$

$\mathrm{Fe}_{3} \mathrm{O}_{4} @ \mathrm{HMSM}$ w/ CTAB

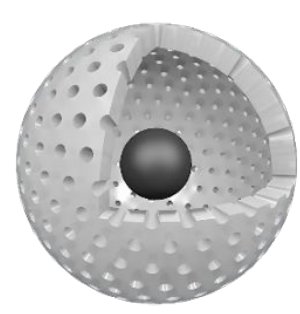

YMSM
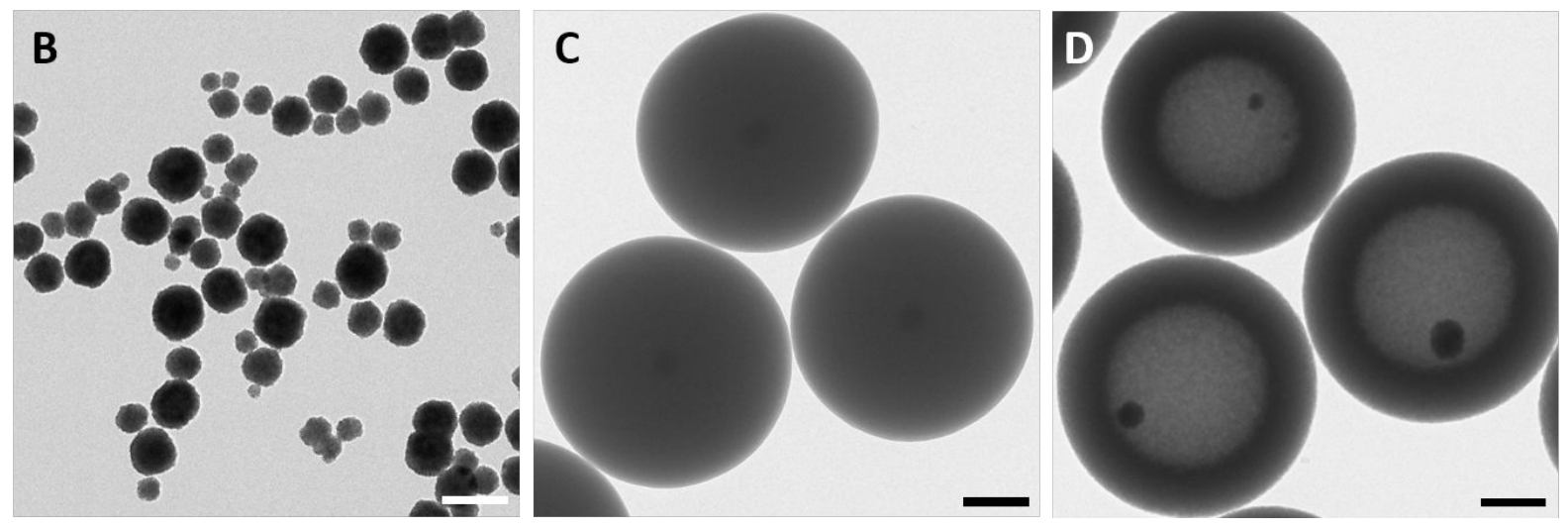

Figure S14. A) Schematic of the self-transformation approach for the synthesis of YMSM in a methanol/water solution. TEM images of $\mathrm{B}$ ) bare $\mathrm{Fe}_{3} \mathrm{O}_{4}$ nanoparticles, C) $\mathrm{Fe}_{3} \mathrm{O}_{4} @ \mathrm{mSiO}_{2}$, and D) YMSMs. Scale bars represent $500 \mathrm{~nm}$. 


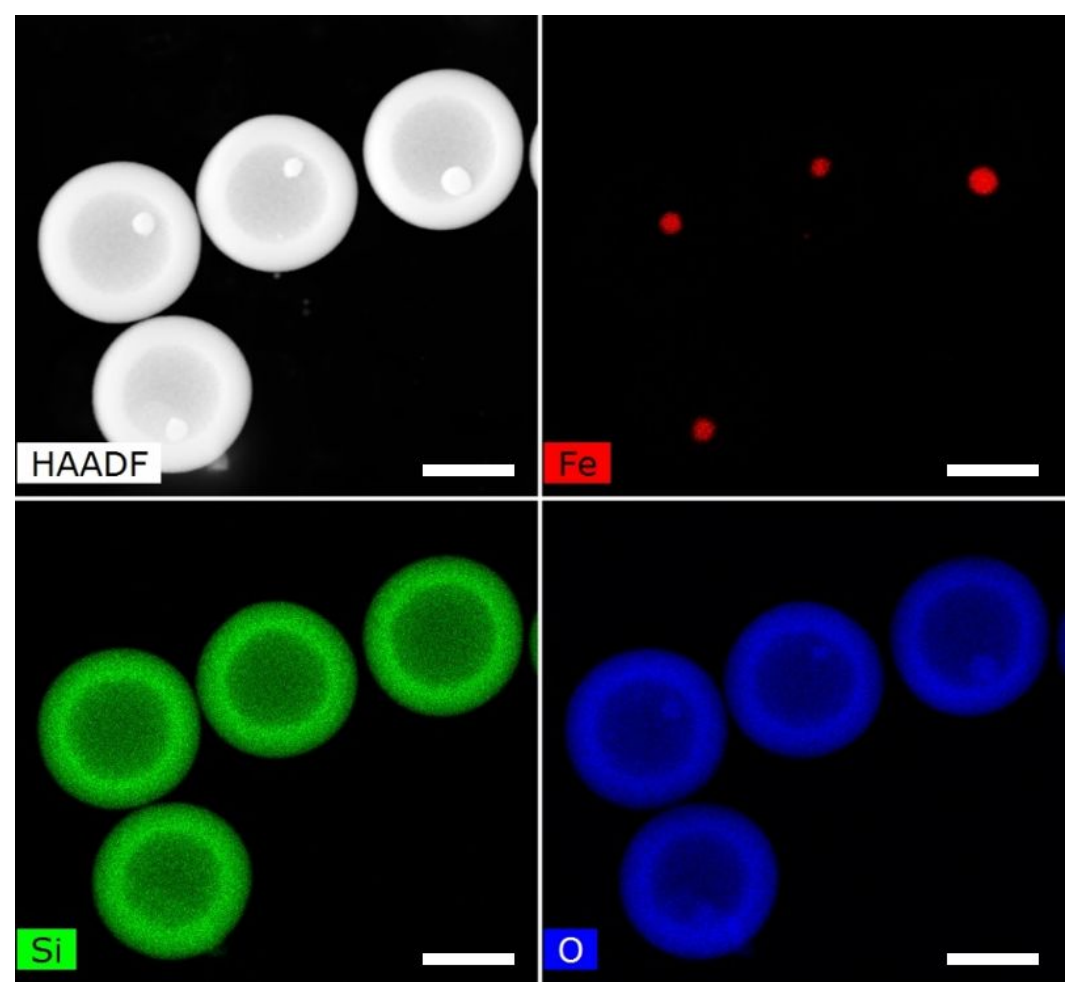

Figure S15. EDX elemental maps of YMSMs. Scale bars represent $1 \mu \mathrm{m}$.

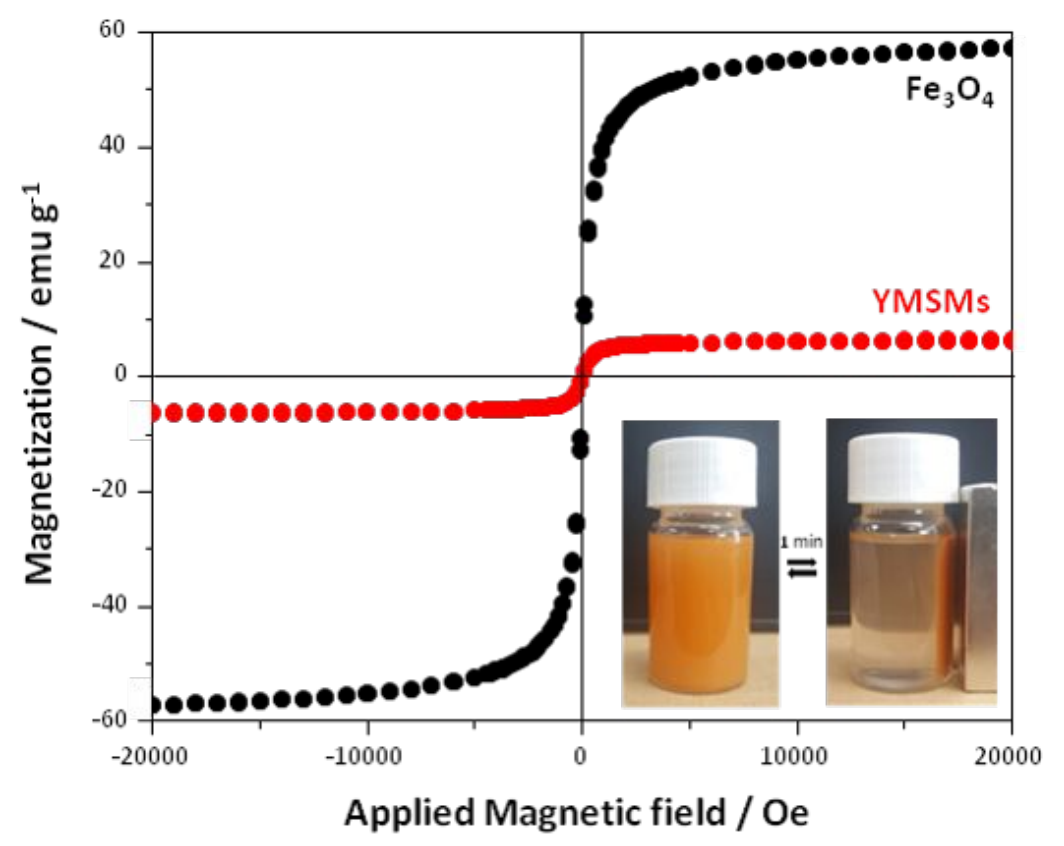

Figure S16. Room-temperature magnetization curves of $\mathrm{Fe}_{3} \mathrm{O}_{4}$ nanoparticles and YMSMs. Inset shows the separation of YMSMs by a magnet. 


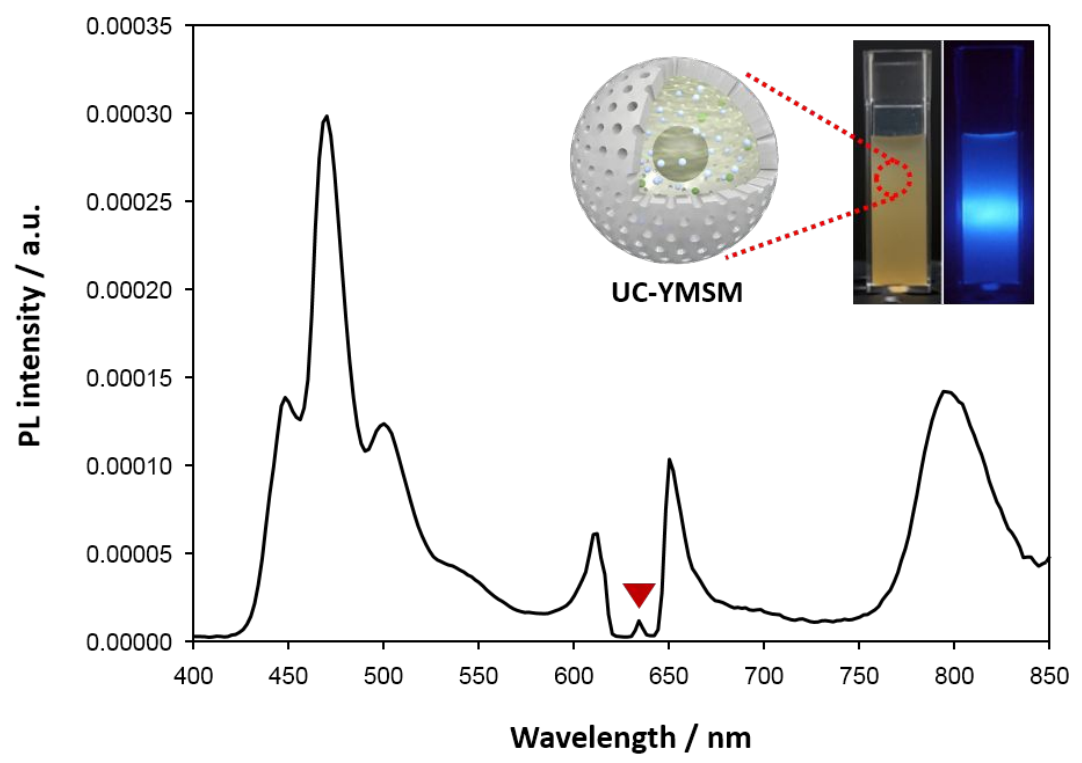

Figure S17. Emission profile and digital images (inset) of UC-YMSMs ([PdTPBP] $=0.43$ $\mathrm{mM}$ and [perylene] $=39.6 \mathrm{mM}$ in encapsulated OA) under $635 \mathrm{~nm}$ laser excitation. The image in (inset, right) was acquired through a $500 \mathrm{~nm}$ short-pass filter.

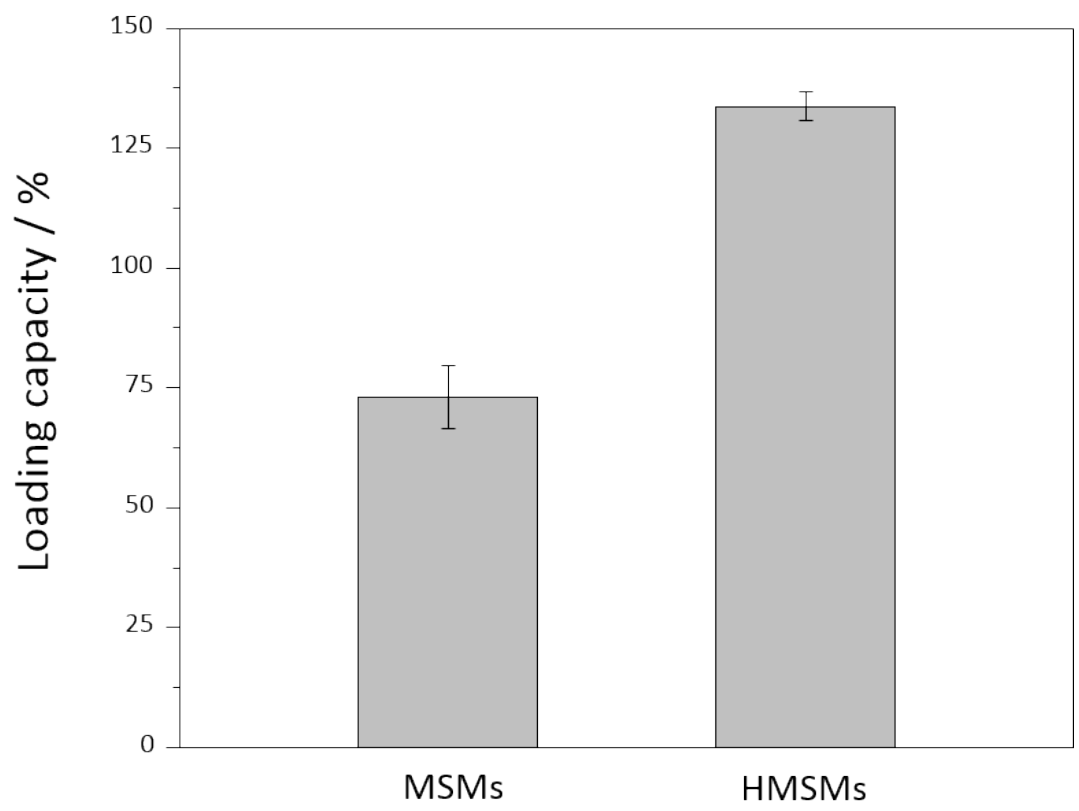

Figure S18. UC oil loading capacity of MSMs and HMSMs synthesized in methanol/water solution. The loading capacity was evaluated by comparing the weight ratio of particles before and after UC oil encapsulation: [(UC oil encapsulated particles - initial particles) / initial particles] $* 100(\%)$. 

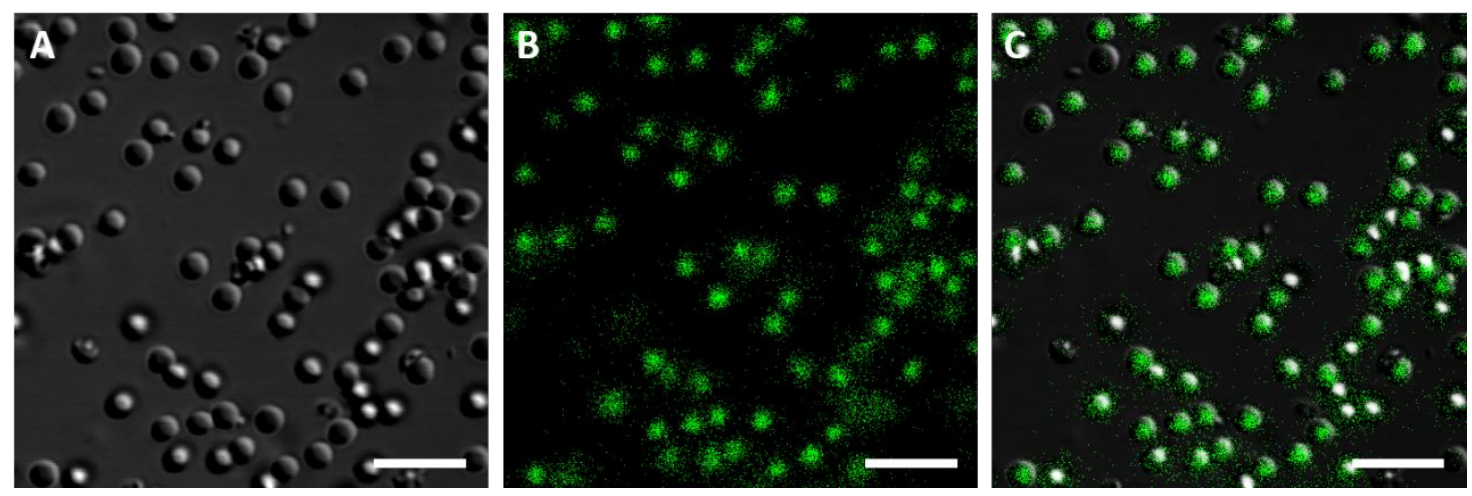

Figure S19. CLSM images of the water suspension of UC-HMSMs ([PdTPBP] $=0.43 \mathrm{mM}$ and $[\mathrm{BPEA}]=26.4 \mathrm{mM}$ in encapsulated OA) under $640 \mathrm{~nm}$ laser excitation; A) bright-field, B) fluorescence channel $(450-550 \mathrm{~nm})$, and C) merged image. Scale bars represent $5 \mu \mathrm{m}$.

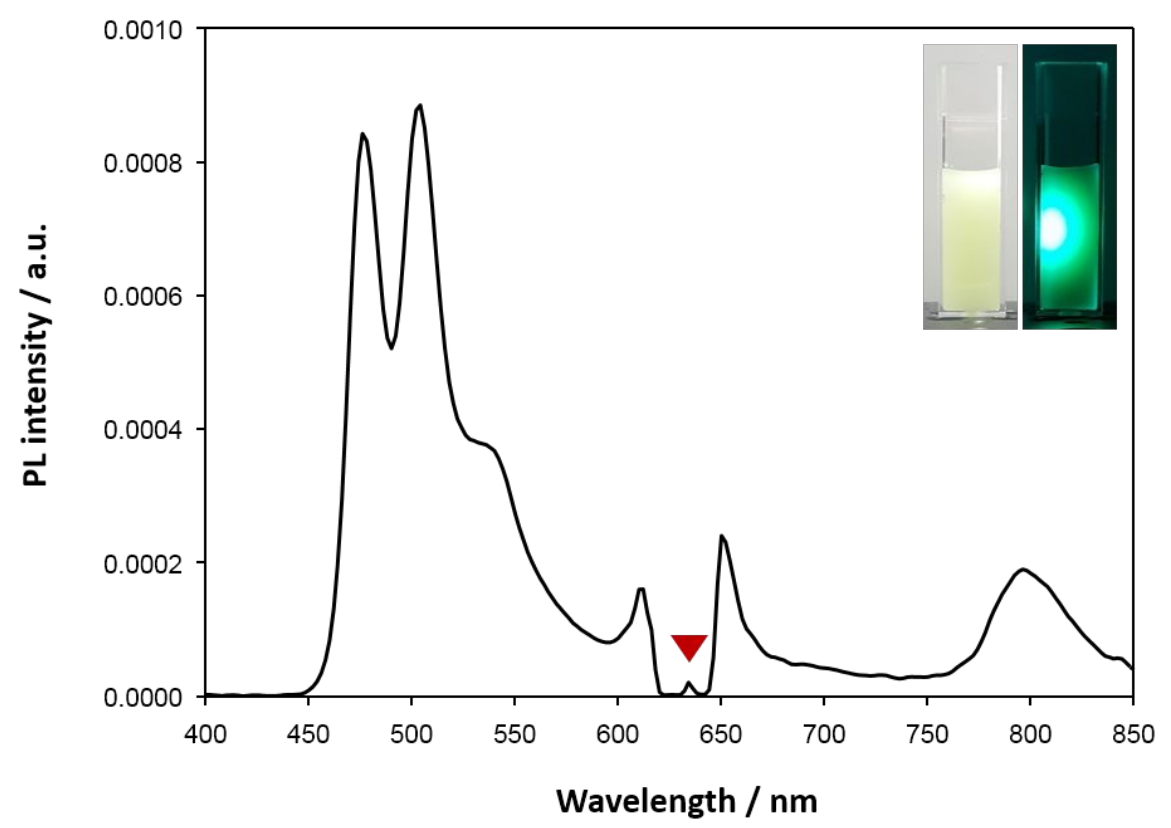

Figure S20. Emission profile and digital images (inset) of UC-HMSMs, containing PdTPBP as sensitizer and BPEA as acceptor $([\mathrm{PdTPBP}]=0.43 \mathrm{mM}$ and $[\mathrm{BPEA}]=26.4 \mathrm{mM}$ in encapsulated OA), under $635 \mathrm{~nm}$ laser excitation. The image in (inset, right) was acquired through a $500 \mathrm{~nm}$ short-pass filter. 


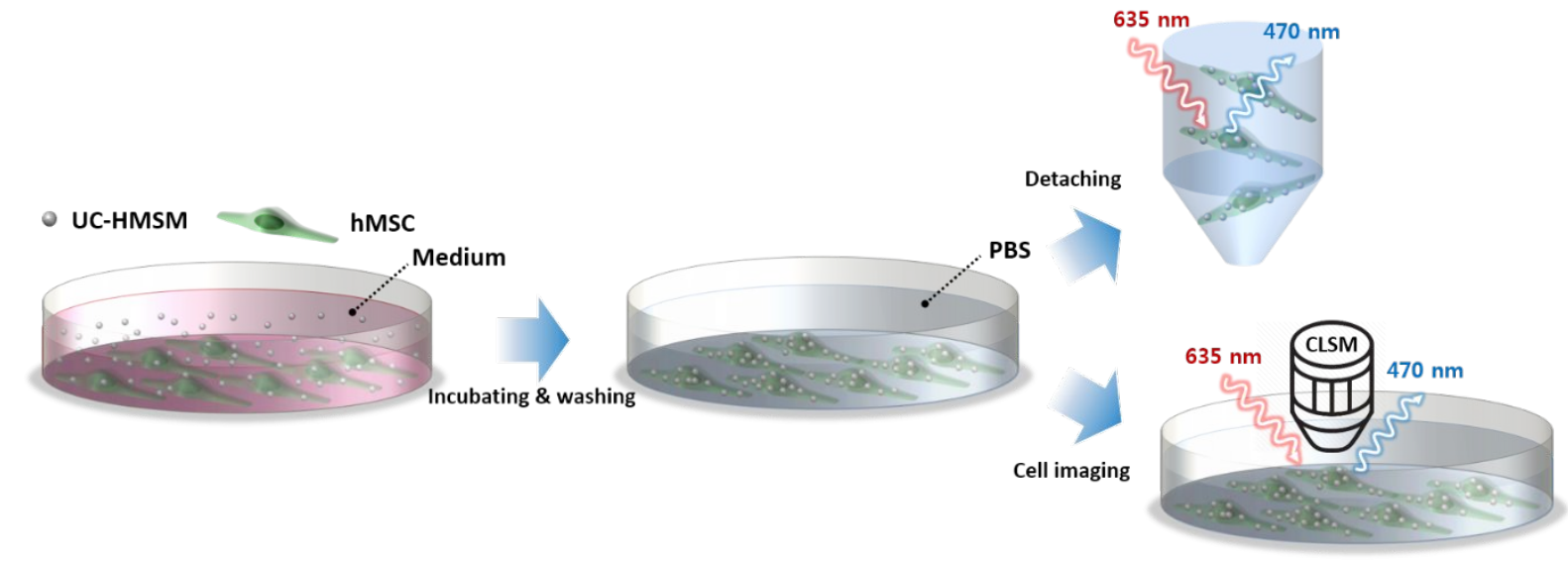

Figure S21. Schematic illustration of the in vitro experiments of cell-mediated TTA-UC system. The hMSCs were cultured in the presence of $200 \mathrm{mg} \mathrm{mL}^{-1}$ of UC-HMSMs for $24 \mathrm{~h}$ and then washed with PBS to obtain UC-hMSCs, followed by TTA-UC test in the Eppendorf tube and CLSM imaging.
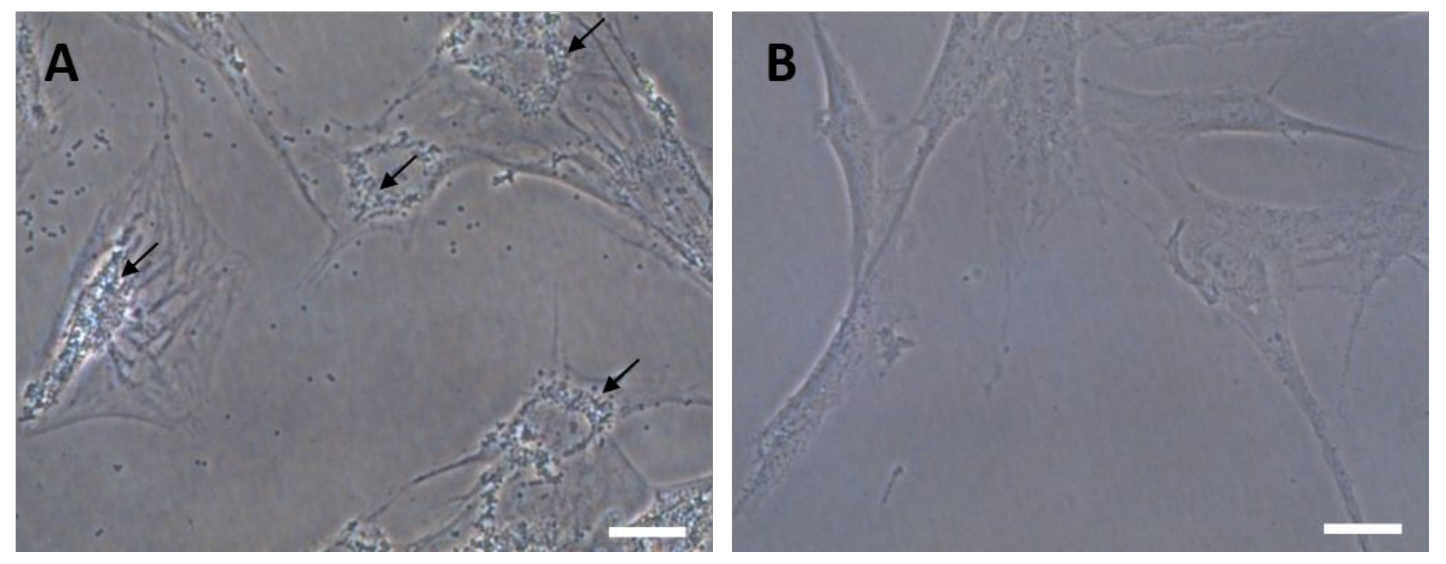

Figure S22. Bright-field images of the hMSCs A) after and B) before incubation with $200 \mu \mathrm{g}$ $\mathrm{mL}^{-1}$ of UC-HMSMs for 24h. Samples were washed seven times with PBS before observation. Scale bars represent $20 \mu \mathrm{m}$. 


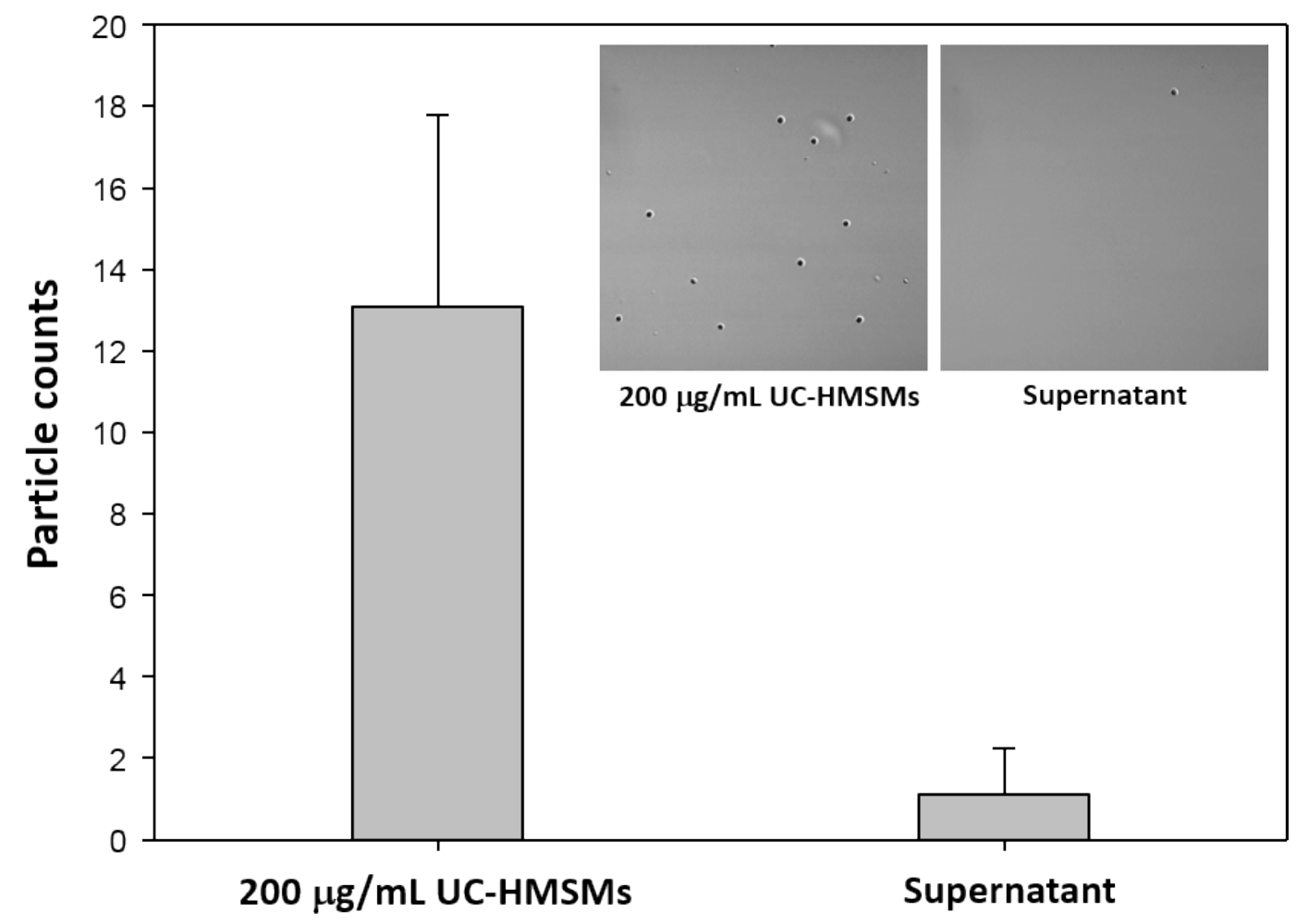

Figure S23. Average particle counts evaluated from bright-field images (100 tiles) of the initial UC-HMSMs suspension $\left(200 \mu \mathrm{g} \mathrm{mL}^{-1}\right)$ and supernatant of the UC-hMSCs suspension. Insets show the representative bright-field images of each sample tile.
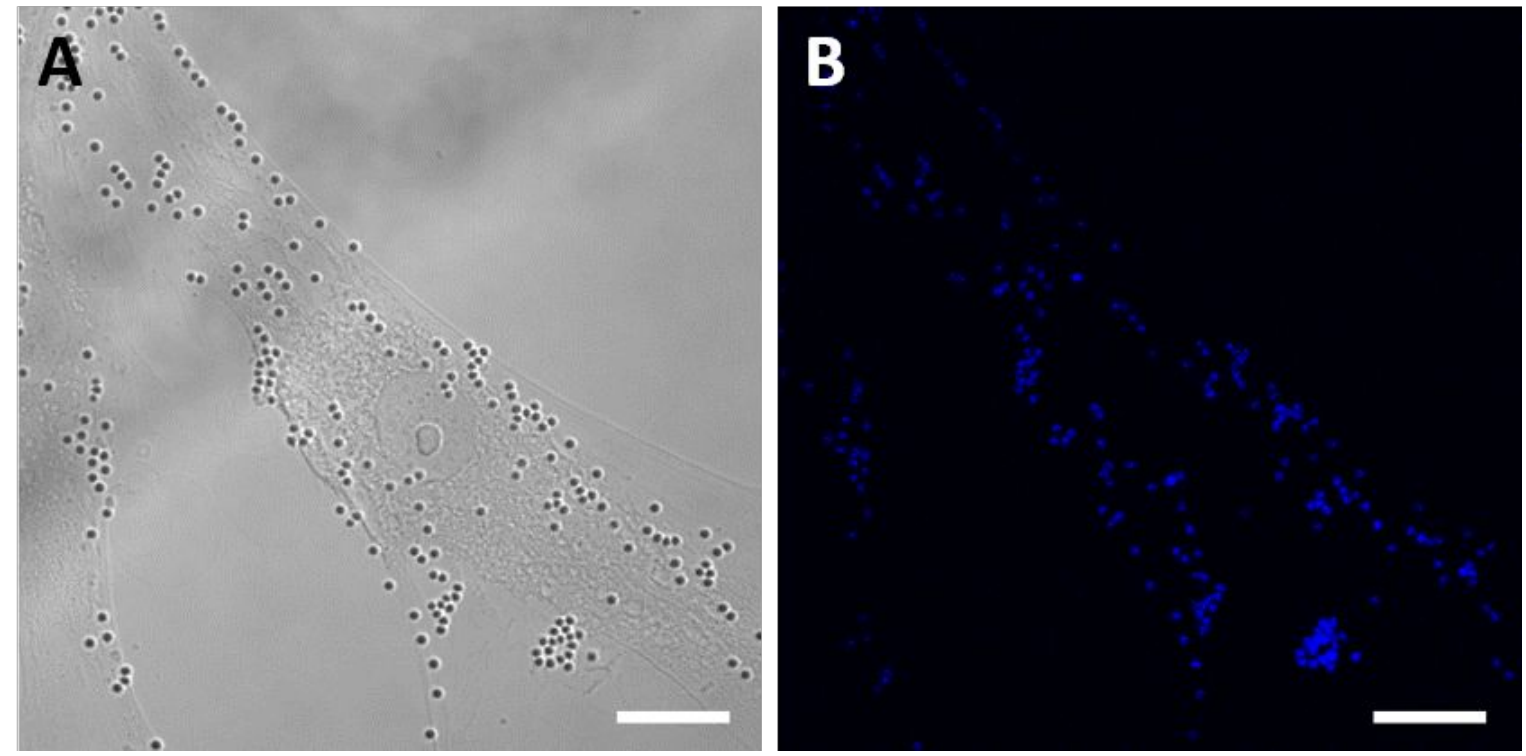

Figure S24. CLSM images of hMSCs incubated with the UC-HMSMs ([PdTPBP] $=0.43 \mathrm{mM}$ and [perylene] $=39.6 \mathrm{mM}$ in encapsulated OA) for $24 \mathrm{~h}$ and then washed with PBS under 640 nm excitation: A) bright-field, and B) fluorescence channel (450-550 nm). 